\title{
Regression after subtotal resection of an optic pathway glioma in an adult without adjuvant therapy: case report
}

\author{
Eveline Teresa Hidalgo, MD, Michelle W. McQuinn, BS, and Jeffrey H. Wisoff, MD \\ Division of Pediatric Neurosurgery, Department of Neurosurgery, NYU Langone Health, New York, New York
}

Optic pathway gliomas (OPGs) are relatively common and benign lesions in children; however, in adults these lesions are nearly always malignant and hold a very poor prognosis. In this report the authors present the case of an adult patient with a benign OPG who underwent subtotal resection without adjuvant therapy and has had no tumor progression for more than 20 years. A 50-year-old woman presented with a 2-year history of personality changes, weight gain, and a few months of visual disturbances. Ophthalmological evaluation showed incomplete right homonymous hemianopsia. MRI demonstrated a $2.5 \times 2.5 \times 2.5-\mathrm{cm}$ enhancing left-sided lesion involving the hypothalamus with extension into the suprasellar cistern, extending along the left optic tract and anterior to the level of the optic chiasm. A biopsy procedure revealed a juvenile pilocytic astrocytoma. A subtotal resection of approximately $80 \%$ of the tumor was performed. Postoperatively, the patient experienced complete resolution of her personality changes, and her weight decreased back to baseline. Ophthalmological examination showed increased right homonymous hemianopsia. In the years following her surgery, there was a spontaneous decrease in tumor size without adjuvant therapy. The patient continues to have an excellent quality of life despite a visual field defect, and no further tumor growth has been observed.

https://thejns.org/doi/abs/10.3171/2017.12.JNS172188

KEYWORDS optic pathway gliomas; progression-free survival; juvenile pilocytic astrocytoma; oncology

$\mathrm{O}$ PTIC pathway gliomas (OPGs) are typically benign, slow-growing lesions that occur in children. ${ }^{5}$ Histologically, OPGs in children are generally welldifferentiated, low-grade gliomas, usually pilocytic astrocytomas, associated with long-term patient survival. ${ }^{1,14,15}$ The treatment options of OPGs in children include observation, radiation therapy, chemotherapy, and surgery. ${ }^{1}$ Observation is suggested for asymptomatic patients with small tumors without mass effect leading to visual symptoms, hydrocephalus, or endocrine involvement. Chemotherapy with carboplatin and vincristine is now commonly used as first-line treatment for symptomatic OPGs in children. ${ }^{1}$

In adults, OPGs are very rare, and are almost exclusively malignant gliomas that often lead to rapid visual loss and are ultimately fatal in almost all cases., ${ }^{4,910,12,14,15}$ The average age at diagnosis of malignant optic gliomas is reported to be 57 years. ${ }^{14}$ Treatment for OPGs in adults has histori- cally been approached in a different manner than children, which is likely due to the malignant nature of the OPGs. Considering the observed poor prognosis of these tumors, limited surgery and/or biopsy are considered the standard of care, followed by adjuvant radiotherapy (in the case of anaplastic astrocytoma) or adjuvant chemo-radiotherapy with temozolomide (in the case of glioblastoma). ${ }^{11,14,15}$ In both children and adults, the indications for primary surgical debulking can include elevated intracranial pressure, hydrocephalus, extrinsic compression of the visual apparatus and neural structures, or progression of endocrine, neurological, or visual symptoms. ${ }^{6,16}$ Gross-total resection is never a goal of the surgery due to the location of the lesion involving the optic pathways.

In this paper we present the case of an adult patient with an OPG in which radiological regression after subtotal resection was observed. Twenty years after surgery, the patient has an excellent quality of life. 


\section{Case Report}

History and Examination

A 50-year-old woman presented with a 2-year history of personality changes and significant weight gain. Shortly before her initial visit, she noted difficulty reading the end of the line, and an ophthalmological evaluation showed incomplete right homonymous hemianopsia.

MRI demonstrated a $2.5 \times 2.5 \times 2.5-\mathrm{cm}$ enhancing lesion along the course of the left optic tract with anterior extension to the level of the optic chiasm (Fig. 1 A1, B1, and $\mathrm{C} 1$ ). There were small cystic components measuring less than $1 \mathrm{~cm}$ in diameter along the superior aspect of this tumor as well as along the anteromedial aspect. The optic nerves anterior to the level of the chiasm were normal in caliber and demonstrated no abnormal enhancement. The right optic tract was normal in appearance. The signal characteristics and enhancing properties were radiologically most consistent with a hypothalamic/chiasmatic OPG. A biopsy of the tumor at a different institution diagnosed a juvenile pilocytic astrocytoma (JPA), and radiation therapy was recommended. The patient presented at our institution for a second opinion, and we recommended subtotal resection to decompress the optic structures and the brain.

\section{Operation}

The patient underwent stereotactic-guided left pterional craniotomy for tumor resection. Intradurally, tumor was identified medial to the proximal middle cerebral artery, which was followed proximally to the carotid artery.

After dissection medial to the carotid artery, the arachnoid of the suprasellar cistern was opened and both optic nerves were identified and appeared normal. The tumor was readily visible, arising from the undersurface and left side of the optic chiasm and optic tract. It was displacing the carotid artery laterally, the left optic nerve and chiasm superomedially, as well as elevating the anterior cerebral artery. The third nerve was then identified, followed by identification of the brain stem.

Working in the left opticocarotid triangle, the tumor was separated from the posterior surface of the carotid artery and from the posterior surface of the carotid bifurcation. The tumor was entered and internally debulked using ultrasonic aspiration and bipolar cautery.

Resection continued until what appeared to be gliotic margin of tumor was identified under stereotactic navigation. At this point, it appeared that the tumor was completely resected from the brain stem. A gliotic plane between the tumor and the optic chiasm and left optic tract was es-

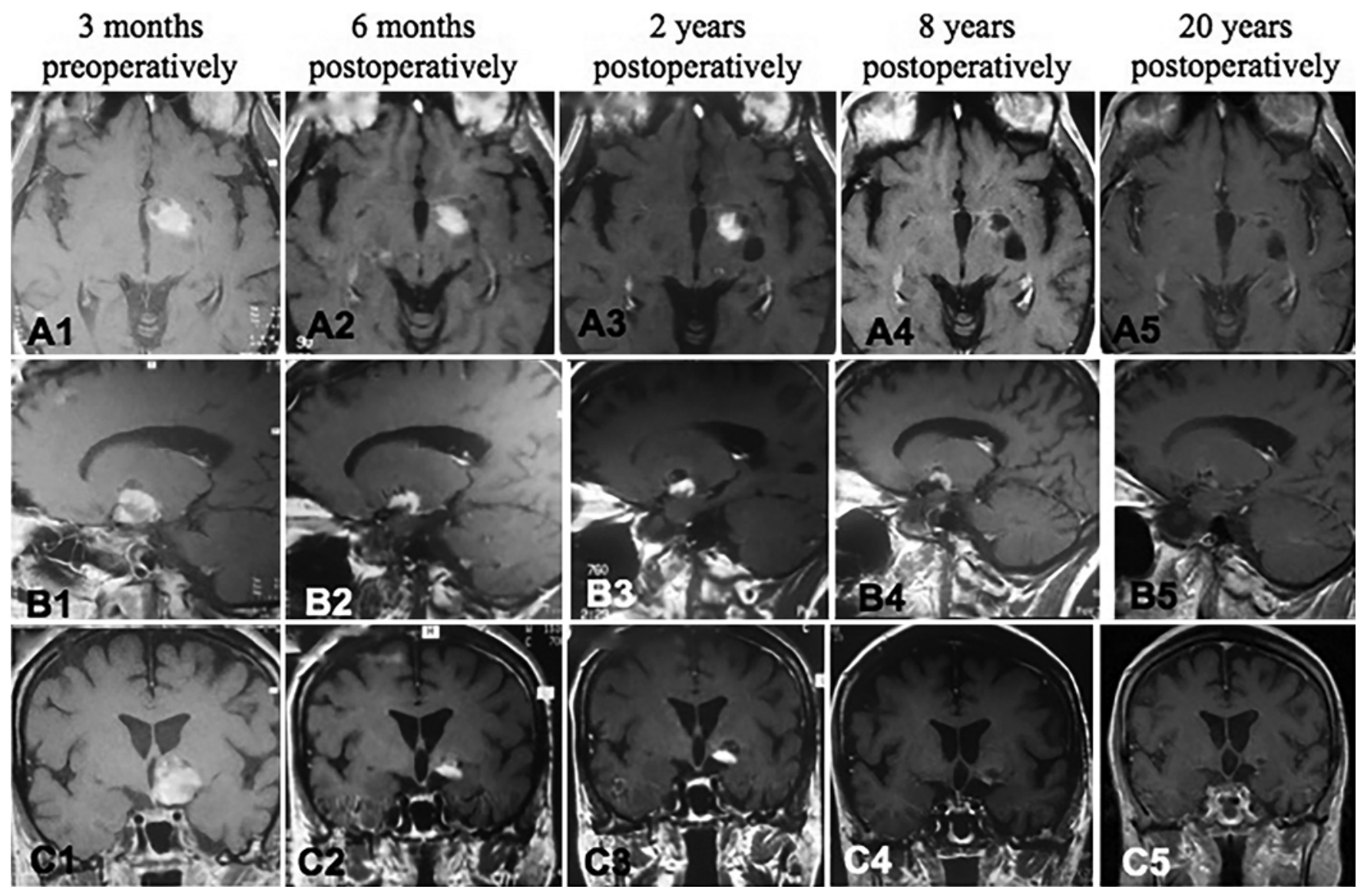

FIG. 1. Contrast-enhanced T1-weighted axial (A1-A5), sagittal (B1-B5), and coronal (C1-C5) brain MRI 3 months preoperatively (A1, B1, and C1), 6 months postoperatively (A2, B2, and C2), 2 years postoperatively ( $A 3, B 3$, and $C 3), 8$ years postoperatively (A4, B4, and C4), and 20 years postoperatively (A5, B5, and C5). 
tablished in order to remove tumor from the lateral aspect of the optic chiasm. Resection was continued deep to the gliotic plane and lateral to the third ventricle. The region was debulked, and the entire capsule was resected from the suprasellar region. The pituitary stalk was intact, and the entire undersurface of the optic chiasm was clear of tumor.

\section{Pathological Findings}

Grossly, tumor samples from the resection appeared as gray-tan soft tissue. Histologically, tumor samples from the resection showed characteristics of a low-grade astrocytoma consistent with pilocytic astrocytoma.

\section{Postoperative Course}

Postoperatively, the patient had transient diabetes insipidus and syndrome of inappropriate antidiuretic hormone secretion, which resolved at the time of discharge and did not require any medications for diabetes insipidus. She did experience an increase in her visual field cut, up to complete right homonymous hemianopsia. A postoperative MR image demonstrated approximately $80 \%$ removal of tumor without any new vascular injury or ischemic damage.

One month following the operation her preexisting psychiatric condition had completely resolved, and her weight had decreased back to baseline, although she continued to have a right visual field cut. Follow-up MR images have been obtained regularly over the past 20 years. Over this period, imaging has revealed spontaneous regression of the tumor in the years following surgery (Fig. 1). There is minimal enhancement in the left optic tract, without significant mass effect. Twenty years following her tumor resection, she continues to have complete right homonymous hemianopsia, but her neurological examination is otherwise unremarkable, and she continues to experience an excellent quality of life without progression on radiographic imaging.

\section{Discussion}

We present the case of a benign OPG in an adult treated with surgery alone that resulted in a favorable outcome, similar to that of a childhood OPG. The indication for subtotal resection in our patient was compression of the visual apparatus and neural structures. The decision not to pursue adjuvant therapy was informed by previous studies that observed the course of pilocytic astrocytomas in both adult and pediatric patients following treatment. In a prospective multiinstitutional study analyzing more than 500 pediatric patients with JPAs, Wisoff et al. reported progression-free survival in over $50 \%$ of patients with residual tumors after subtotal resection at 8 years. ${ }^{17}$ In an adult series, Brown et al. reported a 94\% 5-year progression-free survival rate with supratentorial JPAs that had undergone resection without adjuvant therapy. ${ }^{2}$ In the case of our patient, although she was an adult presenting with an OPG, the histological diagnosis of JPA indicated an outcome more similar to that of childhood OPGs that have a $96.5 \%$ 5-year overall survival rate. ${ }^{7}$ This further supported the decision to forgo the adjuvant therapy that is often recommended to adults with malignant gliomas, and subtotal resection was considered to be sufficient.
Our patient in this case report presented with right homonymous hemianopsia, personality changes, and weight gain. This is an unusual presentation for an adult OPG, as the common presenting factors typically include rapidly progressive visual field deficits and diminishing visual acuity, ultimately progressing to blindness., ${ }^{4,12,14,15}$ In a review of 21 patients, Wabbels et al. found that the time course from onset of visual dysfunction to blindness ranged from 2 weeks to 12 months. ${ }^{15}$

Histological findings in our patient revealed a JPA/ low-grade glioma, which is the most common histological finding in childhood OPGs. ${ }^{1}$ Unfortunately, no tissue was available for further molecular studies at this time due to the length of follow-up. To our knowledge, there have been 8 documented cases of low-grade OPGs in adults that were not fatal within 1 to 2 years. ${ }^{11}$ Of these 8 cases, only 1 was verified histologically as a JPA. This patient's presenting symptoms included visual field impairment as well as blurred vision. ${ }^{11}$ Despite tumor growth, this patient's vision was reported to have remained stable during the follow-up period of 1 year. ${ }^{11}$ All other adult optic gliomas reported in the literature were found to be of malignant pathology. ${ }^{4,9-12,14,15}$ Malignant adult OPGs were classified as either glioblastomas or anaplastic astrocytomas. ${ }^{4,9-11,14,15}$ The different prognosis of patients with JPA compared to patients with glioblastoma or anaplastic astrocytoma likely accounts for the remarkable progression-free survival observed in our patient, as the same tumor in childhood generally leads to a favorable prognosis. ${ }^{1}$

After surgery, the patient developed a new visual field defect, but experienced complete reversal of the preoperative weight gain as well as the personality changes. An unexpected positive result of the surgery was spontaneous regression of the tumor over time. This has been previously described in the literature following subtotal resection without adjuvant therapy. ${ }^{1}$

The spontaneous regression of the tumor observed in our patient was present 8 years after the surgery. Similar findings have been reported in children and young adults with biopsy or partially resected OPG without adjuvant therapy. Regression has been observed early after surgery; ${ }^{8,13}$ however, late regression up to 5 years after surgery in patients with OPG and a histological diagnosis of pilocytic astrocytoma has been reported. ${ }^{3,13}$ Whether this phenomenon represents cell/tumor senescence, apoptosis, disturbance of the tumor microvasculature, or some combination of these and other factors is unclear, and remains speculative. To our knowledge, this clinical and radiographic course has not been described in adults with OPG.

In summary, we report the case of an adult presenting with a benign OPG who was treated similarly to children with OPG. After subtotal resection more than 20 years ago, she has remained progression free with an excellent quality of life.

\section{Acknowledgments}

We thank Prashant Rao for his contribution in drafting the article.

\section{References}

1. Binning MJ, Liu JK, Kestle JR, Brockmeyer DL, Walker 
ML: Optic pathway gliomas: a review. Neurosurg Focus 23(5):E2, 2007

2. Brown PD, Buckner JC, O'Fallon JR, Iturria NL, Brown CA, O'Neill BP, et al: Adult patients with supratentorial pilocytic astrocytomas: a prospective multicenter clinical trial. Int J Radiat Oncol Biol Phys 58:1153-1160, 2004

3. Colosimo C, Cerase A, Maira G: Regression after biopsy of a pilocytic opticochiasmatic astrocytoma in a young adult without neurofibromatosis. Neuroradiology 42:352-356, 2000

4. Dinh TT, Wang YY, Rosenfeld JV, Cherny M: Glioblastoma of the optic chiasm. J Clin Neurosci 14:502-505, 2007

5. Dutton JJ: Gliomas of the anterior visual pathway. Surv Ophthalmol 38:427-452, 1994

6. Goodden J, Pizer B, Pettorini B, Williams D, Blair J, Didi $\mathrm{M}$, et al: The role of surgery in optic pathway/hypothalamic gliomas in children. J Neurosurg Pediatr 13:1-12, 2014

7. Johnson DR, Brown PD, Galanis E, Hammack JE: Pilocytic astrocytoma survival in adults: analysis of the Surveillance, Epidemiology, and End Results Program of the National Cancer Institute. J Neurooncol 108:187-193, 2012

8. Kernan JC, Horgan MA, Piatt JH, D’Agostino A: Spontaneous involution of a diencephalic astrocytoma. Pediatr Neurosurg 29:149-153, 1998

9. Liu Y, Zhang-Nunes S, Zhu X, Xu X, Sun X, Wu Y: Late adult onset optic pathway astrocytoma. J Clin Neurosci 20:1610-1612, 2013

10. Matloob S, Fan JC, Danesh-Meyer HV: Multifocal malignant optic glioma of adulthood presenting as acute anterior optic neuropathy. J Clin Neurosci 18:974-977, 2011

11. Shofty B, Constantini S, Bokstein F, Ram Z, Ben-Sira L, Freedman S, et al: Optic pathway gliomas in adults. Neurosurgery 74:273-280, 2014

12. Spoor TC, Kennerdell JS, Martinez AJ, Zorub D: Malignant gliomas of the optic nerve pathways. Am J Ophthalmol 89:284-292, 1980

13. Takenchi H, Kabuto M, Sato K, Kubota T: Chiasmal gliomas with spontaneous regression: proliferation and apoptosis. Childs Nerv Syst 13:229-233, 1997

14. Traber GL, Pangalu A, Neumann M, Costa J, Weller M,
Huna-Baron R, et al: Malignant optic glioma-the spectrum of disease in a case series. Graefes Arch Clin Exp Ophthalmol 253:1187-1194, 2015

15. Wabbels B, Demmler A, Seitz J, Woenckhaus M, Bloss HG, Lorenz B: Unilateral adult malignant optic nerve glioma. Graefes Arch Clin Exp Ophthalmol 242:741-748, 2004

16. Wisoff JH, Abbott R, Epstein F: Surgical management of exophytic chiasmatic-hypothalamic tumors of childhood. $\mathbf{J}$ Neurosurg 73:661-667, 1990

17. Wisoff JH, Sanford RA, Heier LA, Sposto R, Burger PC, Yates AJ, et al: Primary neurosurgery for pediatric low-grade gliomas: a prospective multi-institutional study from the Children's Oncology Group. Neurosurgery 68:1548-1555, 2011

\section{Disclosures}

The authors report no conflict of interest concerning the materials or methods used in this study or the findings specified in this paper.

\section{Author Contributions}

Conception and design: Hidalgo, Wisoff. Acquisition of data: Hidalgo, McQuinn. Analysis and interpretation of data: all authors. Drafting the article: all authors. Critically revising the article: all authors. Reviewed submitted version of manuscript: all authors. Approved the final version of the manuscript on behalf of all authors: Hidalgo.

\section{Supplemental Information \\ Previous Presentations}

Portions of this work were presented in poster form at the 84th AANS Annual Scientific Meeting on April 30-May 4, 2016, in Chicago, Illinois.

\section{Correspondence}

Eveline Teresa Hidalgo: NYU Langone Health, New York, NY. teresa.hidalgo@nyumc.org. 